\section{Kidney \\ Blood Pressure Research}

\title{
Intrarenal Arterial Lesions Are Associated with Higher Blood Pressure, Reduced Renal Function and Poorer Renal Outcomes in Patients with IgA Nephropathy
}

\author{
Ying Zhang ${ }^{\mathrm{a}}$ Lili Sun ${ }^{\mathrm{a}}$ Suhan Zhou ${ }^{\mathrm{a}}$ Qihe Xu ${ }^{\mathrm{b}}$ Qiannan Xu ${ }^{\mathrm{a}} \quad$ Dongyu Liu ${ }^{\mathrm{a}}$ \\ Lu Liu ${ }^{\mathrm{a}}$ Ruimin $\mathrm{Hu}^{\mathrm{a}}$ Songxia Quan ${ }^{\mathrm{a}}$ Guolan Xing ${ }^{\mathrm{a}}$ \\ aDepartment of Nephrology, First Affiliated Hospital of Zhengzhou University, Zhengzhou, China; \\ bMedical Research Council Centre for Transplantation, King's College London, London, United Kingdom
}

\section{Key Words}

Intrarenal vascular lesions $\bullet$ IgA nephropathy • Outcomes • Arterial fibrotic intimal thickening - Arteriolar hyaline - Endotheliocyte swelling - Arteriolar inflammatory cell infiltration • Arteriolar thrombosis

\begin{abstract}
Background/Aims: Arterial fibrotic intimal thickening and arteriolar hyaline are considered common pathological features in immunoglobulin A nephropathy (IgAN), whereas little is known about the acute pathological manifestations of endothelial cell injury. The aim of this study was to investigate characteristics of intrarenal arterial lesions and to estimate their prognostic values in patients with IgAN. The primary renal endpoint was a $50 \%$ reduction in estimated glomerular filtration rate (eGFR) or end-stage renal disease (ESRD). Methods: Various renal arterial lesions (arterial fibrotic intimal thickening, arteriolar hyaline, arteriolar endotheliocyte swelling, arteriolar inflammatory cell infiltration, and arteriolar thrombosis) in 1683 patients with IgAN were reviewed and reclassified using a semi-quantitative scoring system. Their correlations with clinical features, pathological characteristics, and renal outcomes were evaluated. Results: The prevalence of intrarenal arterial lesions was up to $72.2 \%$ in IgAN patients. There were 978 patients (58.1\%) with arterial fibrotic intimal thickening, 350 patients (20.8\%) with arteriolar hyaline, 432 patients (25.7\%) with arteriolar endotheliocyte swelling, 356 patients (21.2\%) with arteriolar inflammatory cell infiltration and 43 patients (2.6\%) with arteriolar thrombosis. Arterial fibrotic intimal thickening and arteriolar hyaline were strongly associated with higher mean arterial pressure (MAP) and reduced eGFR $(P<0.001)$ but were not related to proteinuria at the time of renal biopsy. In contrast, arteriolar endotheliocyte swelling and arteriolar thrombosis were correlated with heavier proteinuria as well as higher
\end{abstract}




\section{Kidney Blood Pressure Research}

MAP and reduced eGFR. During follow-up, patients with vascular lesions received more renin-angiotensin system (RAS) blockade and less glucocorticoid and showed poorer renal outcomes. Univariate Cox model showed that the presence of renal vascular lesions [hazard ratio $(\mathrm{HR})=25.01,95 \%$ confidence interval $(\mathrm{CI}): 6.19$ to $101.03, P<0.001$ ] was a risk factor for renal outcomes. However, in multivariable Cox analysis, which included clinical factors and the Oxford-MEST-C, vascular lesions were not significantly associated with an increased risk of renal failure. Remarkably, the impact of vascular lesions on the survival from ESRD or $50 \%$ reduction in renal function was eliminated by the use of RAS blockade after adjustment for eGFR, proteinuria, and MAP. Conclusion: Our study demonstrates the high prevalence of vascular lesions, including the chronic and acute arterial pathological changes, in patients with IgAN. The presence of vascular lesions is associated with higher MAP, reduced eGFR and poorer renal outcomes, which could be influenced by the RAS blockade treatment.

(C) 2018 The Author(s)

Published by S. Karger AG, Basel

\section{Introduction}

Immunoglobulin A nephropathy (IgAN) is the most common primary glomerulonephritis throughout the world [1]. The disease is characterized by the deposition predominantly of IgA in the mesangium, whereas histological changes of IgAN on light microscopy may vary greatly among patients. Intrarenal arterial lesions are frequently observed in the biopsies of patients with IgAN. The prevalence of intrarenal arterial wall thickening and arterial hyaline changes was reported in up to $54.6 \%$ of IgAN patients [2].

In contrast to the high prevalence of vasculopathy in IgAN, the characteristics and clinical significance of vascular lesions in IgAN have not been well described. First, most studies focused on the chronic arterial lesions, (such as arterial wall fibrotic thickening and arterial hyaline), whereas acute endothelial cell injury has been rarely studied. Second, the value of vascular lesion as a risk factor for predicting IgAN outcomes is controversial. Intrarenal arterial lesions are candidates for incorporation in the Oxford classification $[3,4]$ as important histological changes. However, the severity of arterial lesions was not significantly associated with decreased survival from ESRD or a $50 \%$ drop in eGFR in the Oxford classification report [4]. In contrast, vasculopathy at the initial biopsy was included as a reliable parameter in several models to estimate renal outcomes in patients with IgAN [5-8].

To clarify these issues, we investigated the acute and chronic vascular lesions in 1683 patients with IgAN. We indicated and graded the severity of vascular lesions using a semi-quantitative scoring system [4] and further assessed their associations with clinical, pathological data and renal outcomes in this large cohort of patients with IgAN. We followed up for a median time of 36 months to explore the details of the relationship between vascular lesions and the prognosis of IgAN.

\section{Materials and Methods}

\section{Patients}

Clinical and renal histopathological data of 1683 patients with renal biopsy-proven IgAN, who were diagnosed between January 2011 and December 2012 from First Affiliated Hospital of Zhengzhou University, were reviewed and reclassified. None of the cases had clinical or histological evidence of systemic lupus erythematosus, Henoch-Schönlein purpura, or liver disease, including liver cirrhosis. Moreover, patients with $<10$ glomeruli and $<6$ vessels in renal biopsy specimens for light microscopic examination were also excluded. 


\section{Kidney Blood Pressure Research}

Zhang et al:: Arterial Lesions in IgA Nephropathy

\section{Evaluation of renal histopathology}

The renal biopsy specimens were examined by immunofluorescence, light microscopy and electron microscopy. Then, these specimens were reviewed and scored by two independent experienced pathologists who were blinded to the patients' clinical data. When disagreements arose, a third reviewer was included to achieve consensus. According to the revised Oxford classification, the mesangial hypercellularity was defined as M0 and M1. Endocapillary hypercellularity was classified as either absent (E0) or present (E1). Segmental glomerulosclerosis was classified as either absent (S0) or present (S1). Tubulointerstitial lesions were graded $\mathrm{T} 0$ for $(0-25 \%)$, T1 for $(25-50 \%)$ and T2 for (>50\%) according to the percentage of cortical area involved by the tubular atrophy or interstitial fibrosis [3]. Crescents were scored as C0 (no crescents), C1(crescents in a least 1 but $<25 \%$ of glomeruli), or C2 (crescents in at least $25 \%$ of glomeruli) [9].

\section{Scores of vascular lesions}

The arterial fibrotic intimal thickening and arteriolar hyaline scores were evaluated on the basis of the definition described in the Oxford classification with some modifications [3]. The semi-quantitative scores of these renal vascular lesions were determined according to the existence or absence of specific vascular lesions: (1) Arterial fibrotic intimal thickening: By comparing the thickness of the intima to that of the media in the same segment of the vessel, the fibrotic intimal thickening was scored as follows: $0=$ normal; $1=$ thickened to less than the thickness of the media; $2=$ thickened to more than the thickness of the media. The highest arterial score was preferred as the arterial fibrotic intimal thickening score of this specimen. (2) Arteriolar hyaline: The semi-quantitative scores of the arteriolar hyaline were adjusted in order to improve the reproducibility. The presence of arteriolar hyaline on the walls of any artery or arteriole was scored as 1 and its absence as 0 .

Although acute lesions (arteriolar endotheliocyte swelling, arteriolar inflammatory cell infiltration and arteriolar thrombosis) were not included in the Oxford classification system, they were still assessed according to an evaluation system used in lupus nephritis [10]: (1) Arteriolar endotheliocyte swelling: $0=$ absence and 1 = presence of endotheliocyte swelling on the walls of any artery or arteriole. (2) Arteriolar inflammatory cell infiltration: $0=$ absence and 1 = presence of any artery or arteriole with inflammatory cell infiltration. (3) Arteriolar thrombosis: $0=$ absence and $1=$ presence of any microthrombosis in the artery or arteriole.

\section{Clinical evaluation}

The clinical data of each patient were collected at the time of renal biopsy, including age, gender, weight, blood pressure, and laboratory data, such as hemoglobin (HGB), serum albumin (ALB), serum creatinine (SCr), serum uric acid (UA), total cholesterol, triglycerides, urinary protein excretion and urinary red blood cells. eGFR was calculated using the CKD-EPI equation [11] in adults and using the Schwartz equation [12] in children. The primary endpoint was defined as survival from a 50\% reduction in renal function, or ESRD.

\section{Statistical analysis}

Statistical analysis was performed using statistical software SPSS 17.0 (SPSS, Chicago, IL). All numerical data were expressed as the mean \pm SD or as median with range. Categorical variables were expressed in percentages. Differences in semi-quantitative data were tested with the Kruskal-Wallis test and the MannWhitney U-test. Differences in quantitative data were compared using the one-way ANOVA test, and the chi-square test was used for categorical data. Bonferroni's correction was used for multiple comparisons. Spearman's rank-order correlation test was performed to analyze the correlation among various parameters. Kaplan-Meier curves were used to analyze patients' renal survival. Univariate survival analysis was carried out using the log-rank test. Multivariate analysis of patient survival was performed using the Cox regression model. The results were expressed as hazard ratio (HR) with 95\% confidence intervals (CI). Statistical significance was considered at $P<0.05$. 


\section{Kidney Blood Pressure Research}

Kidney Blood Press Res 2018;43:639-650

\begin{tabular}{l|l}
\hline DOI: $10.1159 / 000489290$ & (C) 2018 The Author(s). Published by S. Karger AG, Basel
\end{tabular}

Published online: 26 April, 2018

www.karger.com/kb

Zhang et al.: Arterial Lesions in IgA Nephropathy

\section{Results}

Demographic and clinical characteristics of patients with IgAN

The clinical and pathological data of 1683 patients are summarized in Table 1. Among these patients, there were 1008 males and 675 females, with a ratio of $1.5: 1$. At the time of renal biopsy, the median age was 33.0 years (range from 3 to 83 years old). The median arterial pressure (MAP) was $103.3 \mathrm{mmHg}$. There were 1093 patients $(64.9 \%)$ who had a past hypertension history or potentially undiagnosed hypertension, with a blood pressure above the value of 140/90 $\mathrm{mmHg}$ at admission but without a past hypertension history. The percentage of chronic kidney disease stages 1,2, 3, and 4, according to the eGFR values, was $50.2 \%, 19.1 \%, 20.2 \%$ and $10.5 \%$, respectively. The median proteinuria was $2.1 \mathrm{~g}$ per $24 \mathrm{~h}$.

\section{Prevalence of renal vascular lesions in IgAN}

Among the 1683 patients with IgAN, 1215 patients (72.2\%) presented with acute or chronic renal vascular lesions, while 461 cases $(27.4 \%)$ had both lesions. Chronic arterial lesions (arterial fibrotic intimal thickening and arteriolar hyaline) existed in 1010 (60.0\%) biopsies of IgAN patients. Arterial fibrotic intimal thickening was present in 978 patients (58.1\%), among whom 744 patients (44.2\%) were scored as 1 , and 234 patients $(13.9 \%)$ were scored as 2 . Arteriolar hyaline was found in 350 patients (20.8\%). There were 666 patients (39.6\%) with acute renal vascular lesions whom were identified and classified as follows: 432 patients (25.7\%) with arteriolar endotheliocyte swelling, 356 patients $(21.2 \%)$ with arteriolar inflammatory cell infiltration and 43 patients $(2.6 \%)$ with arteriolar thrombosis (Fig. 1).

\section{Correlations between vascular lesions and clinical data}

Arterial fibrotic intimal thickening and arteriolar hyaline were strongly associated with higher MAP and reduced eGFR at the time of renal biopsy $(P<0.001)$. Remarkably, no relationship was found between proteinuria with arterial fibrotic intimal thickening and arteriolar hyaline $(P=0.319$ and 0.530 , respectively). Arterial fibrotic intimal thickening and arteriolar hyaline were also related to older age, heavier weight and higher serum uric acid levels (data not shown). It was unexpected that lower levels of serum cholesterol were observed in patients with arterial fibrotic intimal thickening and arteriolar hyaline $(P<0.001$ and $P=0.005$, respectively) and that levels of serum triglycerides showed the same but not significant tendencies. The correlations between vascular lesions and major clinical data are summarized in Table 2.

Arteriolar endotheliocyte swelling and arteriolar thrombosis were correlated with higher MAP, heavier proteinuria and reduced eGFR at the time of biopsy. Arteriolar inflammatory cell infiltration was only associated with reduced initial eGFR but was not related to blood pressure and proteinuria. No relationships were observed between the acute arterial lesions and age, weight and serum lipid levels. However, arteriolar endotheliocyte swelling,

Table 1. Demographic and clinical data from patients with IgAN. MAP, mean arterial pressure; $\mathrm{SCr}$, serum creatinine; eGFR, estimated glomerular filtration rate; $\mathrm{SD}$, standard deviation

\begin{tabular}{lc}
\hline Characteristics & $\mathrm{n}=1683$ \\
\hline Age (years), median (range) & $33(4-83)$ \\
Gender (male/female) & $1008 / 675$ \\
Hypertension, cases $(\%)$ & $1326(78.8 \%)$ \\
MAP (mmHg), median (range) & $103.3(70.0-218.3)$ \\
Proteinuria $(\mathrm{g} / 24 \mathrm{~h})$, median (range) & $2.1(0.07-28.5)$ \\
Hematuria, cases $(\%)$ & $1220(72.5 \%)$ \\
SCr $(\mu \mathrm{mmol} / \mathrm{L})$, median $($ range) & $86.0(20.0-425.0)$ \\
eGFR $\left(\mathrm{ml} / \mathrm{min}\right.$ per $\left.1.73 \mathrm{~m}^{2}\right)$, median (range) & $89.0(15.6-197.6)$ \\
Hemoglobin $(\mathrm{g} / \mathrm{L})$, mean $\pm \mathrm{SD}$ & $125.5 \pm 22.9$ \\
Serum albumin $(\mathrm{g} / \mathrm{L}), \mathrm{median}($ range) & $36.4(10.1-53.8)$ \\
Serum uric acid $(\mu \mathrm{mmol} / \mathrm{L})$, median (range) & $360.0(22.9-979.0)$ \\
Serum cholesterol $(\mu \mathrm{mmol} / \mathrm{L})$, median (range) & $4.86(2.16-18.75)$ \\
Serum triglyceride $(\mu \mathrm{mmol} / \mathrm{L})$, median (range) & $1.60(0.11-14.80)$ \\
\hline
\end{tabular}




\section{Kidney \\ Blood Pressure Research}

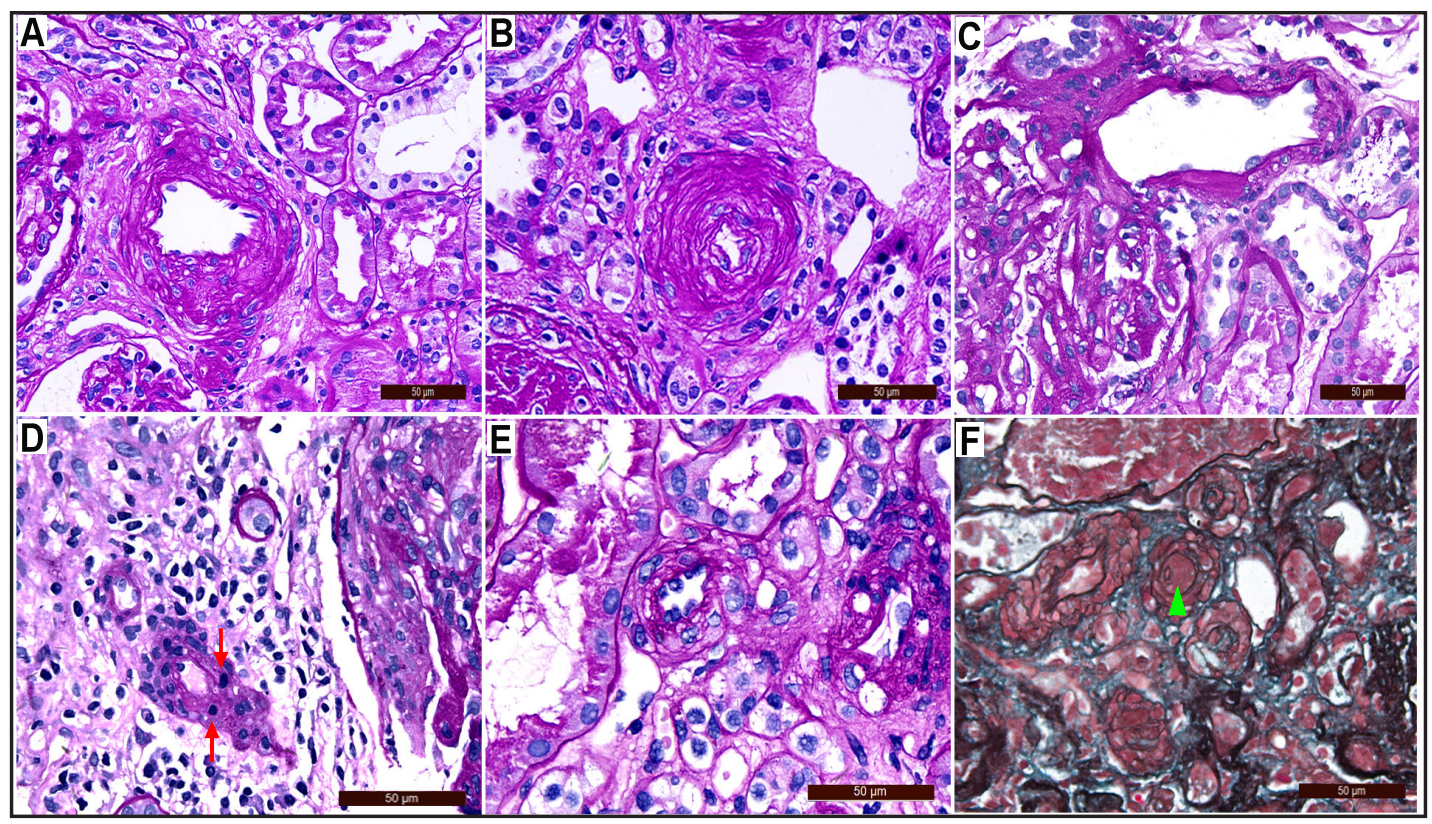

Fig. 1. Arterial lesions in IgA nephropathy. (A) The interlobular artery shows intimal arterial fibrotic intimal thickening and lumen narrowing. The thickness of the intima is less than the thickness of the media in this vessel, thus scoring as 1 (PAS stain). (B) This interlobular artery also shows fibrotic intimal thickening, but the intima has thickened to more than the thickness of the media in this vessel. The fibrotic intimal thickening score for this vessel is 2 (PAS stain). (C) The presence of arteriolar hyaline, shown as homogeneous pink hyaline material, is identified on the walls of this artery (PAS stain). (D) Inflammatory cells (red arrows), stained as blue nucleus by hematoxylin, infiltrate around the walls of this arteriole (PAS stain). (E) Endotheliocyte swelling is presented on the wall of the arteries or arterioles (PAS stain). (F) The arteriole with microthrombosis (green triangle) is shown (trichrome-methenamine stain). Scale bars represent $50 \mu \mathrm{m}$.

Table 2. Correlations between vascular lesions with clinical features at the time of renal biopsy. Data were compared with the Kruskal-Wallis test and the Mann-Whitney U-test. MAP, mean arterial pressure; eGFR, estimated glomerular filtration rate

\begin{tabular}{|c|c|c|c|c|c|c|c|c|}
\hline \multirow{2}{*}{ Variable } & \multicolumn{2}{|l|}{ MAP } & \multicolumn{2}{|l|}{ eGFR } & \multicolumn{2}{|c|}{ Proteinuria } & \multicolumn{2}{|c|}{ Serum cholesterol } \\
\hline & $\mathrm{mmHg}$ & $\mathrm{P}$ & $\mathrm{ml} / \mathrm{min}$ per $1.73 \mathrm{~m}^{2}$ & $\mathrm{P}$ & $\mathrm{g} / 24 \mathrm{~h}$ & $\mathrm{P}$ & $\mu \mathrm{mol} / \mathrm{L}$ & $\mathrm{P}$ \\
\hline \multicolumn{9}{|c|}{ Arterial fibrotic intimal thickening score } \\
\hline 0 & $95.3(70.0-150.0)$ & $<0.001$ & $109.7(24.3-197.6)$ & $<0.001$ & $2.0(0.07-28.5)$ & 0.319 & $5.2(2.5-16.3)$ & $<0.001$ \\
\hline 1 & $110.0(73.3-218.3)$ & & $64.9(15.6-138.6)$ & & $2.2(0.08-16.3)$ & & $4.8(2.2-18.8)$ & \\
\hline 2 & $120.0(70.3-173.3)$ & & $42.6(16.0-153.2)$ & & $2.4(0.31-16.0)$ & & $4.4(2.5-8.2)$ & \\
\hline \multicolumn{9}{|c|}{ Arteriolar hyaline } \\
\hline Absent & $100.0(70.0-218.3)$ & $<0.001$ & $96.8(15.9-197.6)$ & $<0.001$ & $2.1(0.08-28.5)$ & 0.530 & $4.9(2.2-18.8)$ & 0.005 \\
\hline Present & $113.0(73.3-193.3)$ & & $59.1(15.6-153.2)$ & & $2.2(0.07-16.0)$ & & $4.8(2.7-11.8)$ & \\
\hline \multicolumn{9}{|c|}{ Arteriolar endotheliocyte swelling } \\
\hline Absent & $103.0(70.0-193.3)$ & $<0.001$ & $90.9(15.6-197.6)$ & $<0.001$ & $2.1(0.07-18.1)$ & $<0.001$ & $4.8(2.2-18.8)$ & 0.752 \\
\hline Present & $103.3(75.3-218.3)$ & & $83.3(15.7-153.2)$ & & $2.9(0.09-28.5)$ & & $5.0(2.8-16.1)$ & \\
\hline \multicolumn{9}{|c|}{ Arteriolar inflammatory cell infiltration } \\
\hline Absent & 103.7 (70.0-218.3) & 0.422 & $87.8(15.6-197.6)$ & 0.001 & $2.1(0.07-28.5)$ & 0.261 & $4.8(2.2-18.8)$ & 0.462 \\
\hline Present & $100.3(75.0-159.7)$ & & $95.0(15.7-190.0)$ & & $2.1(0.15-13.6)$ & & $5.2(3.0-14.6)$ & \\
\hline \multicolumn{9}{|c|}{ Arteriolar thrombosis } \\
\hline Absent & $102.3(70.0-218.3)$ & $<0.001$ & $90.0(15.7-197.6)$ & $<0.001$ & $2.1(0.07-28.5)$ & 0.001 & $4.9(2.2-18.8)$ & 0.114 \\
\hline Present & $106.7(104.3-173.3)$ & & $33.2(15.6-61.0)$ & & $3.5(1.0-5.5)$ & & $4.4(3.0-7.6)$ & \\
\hline
\end{tabular}




\section{Kidney Blood Pressure Research}

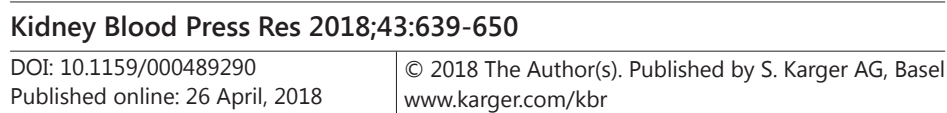

Zhang et al.: Arterial Lesions in IgA Nephropathy

Table 3. Correlations between vascular lesions with pathologic variables of the Oxford classification. P values were calculated by Spearman's rank-order correlation test. M, mesangial hypercellularity; E, endocapillary hypercellularity; S, segmental glomerulosclerosis; T, tubular atrophy or interstitial fibrosis; C, crescents

\begin{tabular}{|c|c|c|c|c|c|c|c|c|c|c|}
\hline \multirow{2}{*}{ Variable } & \multicolumn{2}{|c|}{ M } & \multicolumn{2}{|c|}{$\mathrm{E}$} & \multicolumn{2}{|c|}{$\mathrm{S}$} & \multicolumn{2}{|c|}{$\mathrm{T}$} & \multicolumn{2}{|c|}{$\mathrm{C}$} \\
\hline & $\mathrm{r}$ & $P$ & $\mathrm{r}$ & $P$ & $\mathrm{r}$ & $P$ & $\mathrm{r}$ & $P$ & $\mathrm{r}$ & $P$ \\
\hline Arterial fibrotic intimal thickening & 0.10 & $<0.001$ & -0.03 & 0.229 & 0.33 & $<0.001$ & 0.50 & $<0.001$ & 0.11 & $<0.001$ \\
\hline Arteriolar hyaline & -0.04 & 0.083 & -0.10 & $<0.001$ & 0.13 & $<0.001$ & 0.21 & $<0.001$ & -0.05 & 0.052 \\
\hline Arteriolar endotheliocyte swelling & 0.13 & $<0.001$ & 0.04 & 0.097 & 0.07 & 0.004 & 0.18 & $<0.001$ & 0.08 & 0.001 \\
\hline Arteriolar inflammatory cell infiltration & 0.03 & 0.301 & 0.04 & 0.15 & 0.09 & $<0.001$ & 0.09 & $<0.001$ & 0.08 & 0.001 \\
\hline Arteriolar thrombosis & 0.05 & 0.048 & 0.03 & 0.27 & 0.09 & 0.001 & 0.26 & $<0.001$ & 0.09 & $<0.001$ \\
\hline
\end{tabular}

Table 4. Therapy received during follow-up in relation to vascular lesions. MAP, mean arterial pressure; eGFR, estimated glomerular filtration rate

\begin{tabular}{|c|c|c|c|c|c|c|}
\hline \multirow{2}{*}{ Variable } & \multicolumn{2}{|c|}{ RAS blockade } & \multicolumn{2}{|c|}{ Glucocorticoids } & \multicolumn{2}{|c|}{ Immunosuppression } \\
\hline & $\%$ & $\mathrm{P}$ & $\%$ & $P$ & $\%$ & $P$ \\
\hline \multicolumn{7}{|c|}{ Chronic arterial lesions } \\
\hline Absent & 56.5 & 0.018 & 67.9 & $<0.001$ & 32.9 & 0.113 \\
\hline Present & 63.7 & & 43.7 & & 37.6 & \\
\hline \multicolumn{7}{|c|}{ Arterial fibrotic intimal thickening score } \\
\hline 0 & 57.3 & 0.094 & 66.1 & $<0.001$ & 32.7 & 0.093 \\
\hline 1 & 64.0 & & 46.4 & & 36.9 & \\
\hline 2 & 61.6 & & 35.9 & & 42.1 & \\
\hline \multicolumn{7}{|c|}{ Arteriolar hyaline } \\
\hline Absent & 60.3 & 0.509 & 58.1 & $<0.001$ & 34.9 & 0.261 \\
\hline Present & 62.8 & & 36.4 & & 39.0 & \\
\hline \multicolumn{7}{|c|}{ Acute arterial lesions } \\
\hline Absent & 62.6 & 0.133 & 51.7 & 0.178 & 36.2 & 0.702 \\
\hline Present & 58.0 & & 55.9 & & 35.0 & \\
\hline \multicolumn{7}{|c|}{ Arteriolar endotheliocyte swelling } \\
\hline Absent & 63.4 & 0.001 & 52.5 & 0.397 & 35.9 & 0.824 \\
\hline Present & 52.1 & & 55.6 & & 34.9 & \\
\hline \multicolumn{7}{|c|}{ Arteriolar inflammatory cell infiltration } \\
\hline Absent & 59.1 & 0.048 & 52.6 & 0.473 & 35.1 & 0.549 \\
\hline Present & 66.1 & & 55.2 & & 37.3 & \\
\hline \multicolumn{7}{|c|}{ Arterial thrombosis } \\
\hline Absent & 61.6 & 0.005 & 54.0 & 0.009 & 36.0 & 0.262 \\
\hline Present & 35.5 & & 29.0 & & 25.8 & \\
\hline
\end{tabular}

arteriolar thrombosis and arteriolar inflammatory cell infiltration were all associated with uric acid levels $(P<0.001$ and $P=0.005$, respectively $)$.

\section{Correlations between vascular lesions and pathological data}

Correlations between vascular lesions and the pathologic variables of the Oxford classification are shown in Table 3. Although many of the vascular lesions showed a significant correlation with MEST-C lesions, most of the correlation coefficients were below 0.1 . However, arterial fibrotic intimal thickening was closely linked to segmental sclerosis, interstitial fibrosis and tubular atrophy, suggesting a common pathogenesis.

\section{Impact of vascular lesions on therapy during follow-up}

The use of three majortreatments, RAS blockade, glucocorticoidsandimmunosuppression was assessed in relation to various vascular lesions (Table 4). Patients with arterial fibrotic intimal thickening and arteriolar hyaline received more ACEI/ARB than patients without these lesions (63.7\% versus $56.5 \%$, respectively, $P=0.018$ ). Conversely, glucocorticoids were used to treat patients with arterial fibrotic intimal thickening and arteriolar hyaline less frequently than patients without such findings $(43.7 \%$ versus $67.9 \%$, respectively, $P<$ 0.001). Patients with arteriolar endotheliocyte swelling, inflammatory cell infiltration and 


\section{Kidney \\ Blood Pressure Research}
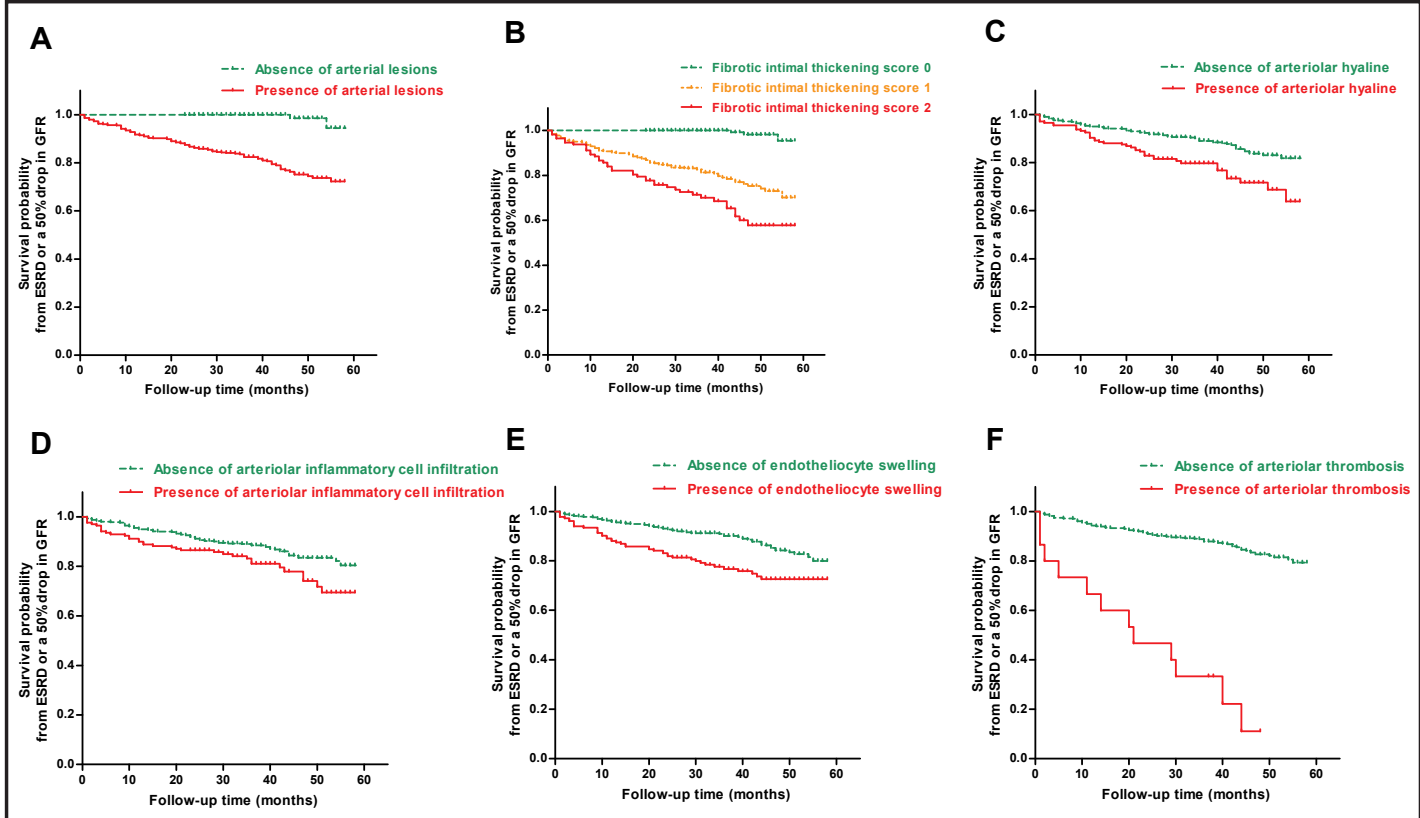

Fig. 2. Survival probability from ESRD or a $50 \%$ drop of eGFR in IgAN patients with and without various renal arterial lesions. (A) The solid line represents patients with any renal arterial lesions. The dashed line presents patients without any renal arterial lesions. Compared to the patients without vascular lesions, the patients with vascular lesions have a significantly lower survival from ESRD or a 50\% drop in eGFR. (B) Compared to the patients without fibrotic intimal thickening, the patients with arterial fibrotic intimal thickening, either mild or severe, have poorer outcomes. (C) Compared to the patients without arteriolar hyaline (the dashed line), the patients with arteriolar hyaline (the solid line) show poorer survival from the endpoint as well as arteriolar inflammatory cell infiltration, (D) endotheliocyte swelling (E), and arteriolar thrombosis $(\mathrm{F})$.

thrombosis had an equal chance to receive glucocorticoids $(\mathrm{P}=0.178)$ as those without acute arterial lesions as well as RAS blockade $(\mathrm{P}=0.133)$. No significant correlations between all types of vascular lesions with immunosuppressive treatment were found $(P>0.05)$.

\section{Correlations between vascular lesions and outcome}

We collected follow-up clinical features and the therapeutic schedule of 1439 (85.5\%) patients. The average length of follow-up was 40 months. During the follow-up period, 244 $(17.0 \%)$ of the patients progressed to the primary endpoint, and two patients died of severe pulmonary infection. Patients without vascular lesions had a significantly higher survival rate from ESRD or a 50\% drop in GFR than patients with any vascular lesion $(99.0 \%$ versus $77.6 \%, P<0.001$ ). Fig. 2 shows the differences in the primary endpoint for the presence and absence of vascular lesions. Additional details about the correlations between vascular lesions and outcome are summarized in Table 5. By univariate analysis, the presence of renal vascular lesions was a risk factor for survival without ESRD or $50 \%$ reduction in initial eGFR in IgAN (HR $=25.01,95 \%$ CI: 6.19 to $101.03, P<0.001$ ). Not only chronic arterial lesions (HR $=28.91,95 \%$ CI: 9.21 to $90.79, P<0.001)$ but also acute arterial lesions $(\mathrm{HR}=2.01,95 \% \mathrm{CI}$ : 1.44 to $2.83, P<0.001$ ) at the time of biopsy predicted a worse outcome in IgAN patients.

For the multivariable Cox analysis, in the first multivariable model, which considered the other pathology variables (MEST-C), the presence of vascular lesions was an independent risk factor for predicting long-term renal survival (HR $=5.51,95 \% \mathrm{CI}: 1.33$ to $22.89, P=$ 0.019 ). The second model was designed to take initial clinical data (eGFR, proteinuria and MAP) into account, except for the pathology data. In this model, vascular lesion was not an independent risk predictor of unfavorable renal outcomes $(P=0.516)$. Remarkably, the 


\section{Kidney \\ Blood Pressure Research}

Table 5. Correlations between vascular lesions and outcomes. Survival rates (\%) and P values were calculated by Kaplan-Meier curves using the log-rank test. HR and CI were calculated from the Cox regression model. ESRD, end stage renal disease; eGFR, estimated glomerular filtration rate; HR, hazard ratio; CI, confidence interval

\begin{tabular}{lccc}
\hline \multirow{2}{*}{ Variable } & \multicolumn{3}{c}{ Survival from ESRD or 50\% drop in GFR } \\
& Survival rate (\%) & P & Univariate HR (95\%CI) \\
\hline Arterial fibrotic intimal thickening score & & & \\
0 & $99.0 \%$ & & \\
1 & $76.2 \%$ & & \\
2 & $62.4 \%$ & & \\
& & & \\
Arteriolar hyaline & & & \\
Absent & $85.5 \%$ & & \\
Present & $74.6 \%$ & & \\
Arteriolar endotheliocyte swelling & & & \\
Absent & & & \\
Present & $86.5 \%$ & & \\
Arteriolar inflammatory cell infiltration & $72.4 \%$ & & \\
Absent & & & \\
Present & & & \\
Arteriolar thrombosis & $84.6 \%$ & & \\
Absent & $77.6 \%$ & & \\
Present & & & \\
\end{tabular}

relationship between vascular lesions and ESRD or 50\% reduction in renal function was evident in those who did not receive RAS blockade (HR $=4.71,95 \%$ CI: 1.46 to $15.22, P=$ $0.010)$ and was lost in those who did $(P=0.334)$ after adjustment for eGFR, proteinuria, and MAP.

\section{Discussion}

Vascular lesions are common pathological characteristics in the renal biopsies of patients with IgAN. Whether the vascular lesions have prognostic significance in IgAN is controversial. In addition, little attention has been paid to acute pathological manifestations of endothelial cell injury, such as arteriolar endotheliocyte swelling, arteriolar thrombosis and arteriolar inflammatory cell infiltration. In this study, we investigated both chronic and acute arterial lesions in a large cohort of patients with IgAN, and explored their correlations with higher blood pressure and reduced renal function. Our data confirmed that renal vascular lesions were a risk factor for unfavorable renal outcomes in IgAN. However, vascular lesions were not an independent risk predictor of renal outcomes when adjusted for initial clinical data and other pathology variables.

In our study, arterial fibrotic intimal thickening and arteriolar hyaline were found in $60.0 \%$ of patients with IgAN, which was higher than previously reported by the Oxford classification [4] and similar to a previous study made by Chinese General Hospital of PLA [2]. One explanation might be that the prevalence and severity of IgAN are higher in China than in non-Asian countries [13]. However, the majority of patients in the original Oxford classification were of European origin. Moreover, the Oxford study excluded patients with an estimated GFR of less than $30 \mathrm{ml} / \mathrm{min} / 1.73 \mathrm{~m}^{2}$, who had high prevalence of vascular lesions [4].

Acute endotheliocyte injury was rarely investigated in patients with IgAN. We found that $25.7 \%$ of IgAN patients presented with arteriolar endotheliocyte swelling, and $2.6 \%$ of IgAN patients had arteriolar thrombosis, which are acute presentations of thrombotic microangiopathy (TMA). It was reported that $53.1 \%$ of patients with IgAN presented with 


\section{Kidney Blood Pressure Research}

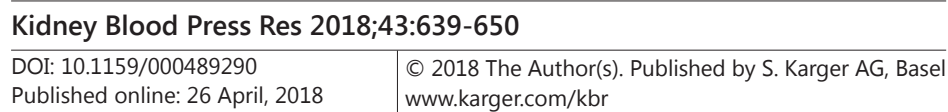

Zhang et al.: Arterial Lesions in IgA Nephropathy

TMA lesions [14], but the study did not describe the acute and organized TMA. In contrast, another study only identified morphologic lesions of TMA in $1.4 \%$ of their patients with IgAN [15]. Our study confirmed a comparable high frequency of acute endotheliocyte injury in IgAN patients and explored their correlation to MAP, eGFR and proteinuria. Chang et al. reported that a TMA injury can be associated with severe proteinuria and usually found in advanced stages of IgAN [16]. Although the acute lesions are reversible, they may in part be attributable to the poor outcomes in patients with these lesions.

Previous study suggested a TMA injury presented in IgAN may be a cause or a consequence of the severe hypertension encountered in most of the patients [16]. We also demonstrated a close association between the TMA lesions with higher blood pressure. Endothelial cell injury caused by mechanical stretch provides a possible explanation for the frequent TMA lesions in patients with IgAN. Accumulating evidences have confirmed that complement activation is involved in the pathogenesis of endothelial damage [17-19]. Complement activation plays an essential role in the progression of IgAN [20, 21]. Future works will need to address the impact of complement activation on the pathogenesis of TMA lesions in patients with IgAN.

Our study confirmed that arterial fibrotic intimal thickening and arteriolar hyaline were strongly associated with initial MAP and eGFR but had no relation with initial proteinuria. These results are similar to previous studies [2, 4]. Higher MAP and lower eGFR were reported as major risk factors for renal outcomes $[6,22,23]$. Compared to patients without vascular lesions, those patients with vascular lesions, who had higher MAP and lower eGFR, probably had poor renal outcomes. The initial proteinuria of patients with and without chronic vascular lesions was comparable; this finding was different from some previous reports [2, 15], and consistent with the Oxford study [4]. It is well known that hypertension can cause atherosclerosis and arteriolar hyaline, while ischemic vascular injury exhibits little proteinuria. This reason may explain the uncorrelated proteinuria with arterial fibrotic intimal thickening and arteriolar hyaline in our data.

The causes of vascular lesions are uncertain in IgAN. Although most patients with atherosclerosis exhibited hypertension, $21.2 \%$ of patients with atherosclerosis still had normal blood pressure. Vascular lesions in these patients could not be explained by the hemodynamic changes induced by hypertension. It is of note that patients with vascular lesions showed a higher percentage of segmental glomerulosclerosis and higher scores of tubular atrophy/interstitial fibrosis in our study. These correlations may suggest a common pathogenesis. Vascular lesions may be considered part of the renal interstitial histopathologic lesions in IgAN. This gives a reasonable explanation of the cause of vascular lesions in the patients without hypertension. Moreover, segmental glomerulosclerosis and tubular atrophy/interstitial fibrosis have been demonstrated to be independent predictive factors of renal outcomes [6,24]. Correlations between vascular lesions with segmental glomerulosclerosis and tubular atrophy/interstitial fibrosis probably also indicated poor renal outcomes in the patients with vascular lesions.

In addition to hemodynamic changes caused by hypertension, hyperlipidemia is also an important cause of atherosclerosis. However, compared to the serum cholesterol and serum triglyceride levels in patients without vascular lesions in this study, neither of these levels was higher in patients with vascular lesions. Thus, hyperlipidemia was likely not a major cause of atherosclerosis in the patients with IgAN. Today, it is believed that atherosclerosis involves a maladaptive inflammatory response [25]. Several inflammatory cytokines take part in the development and progression of IgAN [26, 27]. Further research is necessary to clarify the precise roles of cytokines in the pathogenesis of intimal fibrosis in patients with IgAN.

We demonstrated that compared with patients without vascular lesions in the univariate analysis, patients in the vascular lesions group showed significantly poorer renal outcomes. While in the multivariate model, the presence of vascular lesions was not an independent risk factor for the primary renal endpoint. It is likely that many of the variables (MAP, eGFR, 


\section{Kidney \\ Blood Pressure Research}

tubular atrophy, etc.) are correlated with vascular lesions on biopsy. In addition, there was significant interaction between tubular atrophy and vascular lesions, and the interaction reduced the predictive value of vascular lesions. Hence, renal vascular lesions were not an independent predictor for patients with IgAN.

Of note, the relationship between vascular lesions and poorer renal outcomes was eliminated by the use of RAS blockade. It was reported that RAS blockade increased lumen diameter values, reduced wall thickness and improved endothelium function in a rodent chronic kidney disease model [28]. This evident effect of RAS blockade on the endothelial damage is also possibly reasonable in patients with IgAN. RAS components are demonstrated to be locally synthesized and involved in tissue injury in the disease [29-31]_ENREF_20. Activated intrarenal reactive oxygen species along with increased angiotensinogen were confirmed in patients with IgAN [32], which prompted a potential role of intrarenal RAS axis and oxidative stress in the pathogenesis of arterial lesions of IgAN. These pieces of evidence strongly suggest that vascular injury could possibly be altered by the use of RAS blockade, and give an explanation of the protective effect of RAS blockade in our data.

Concerns were raised that the relationship between vascular lesions and a combined event was influenced by the use of RAS blockade. In patients who received no RAS blockade, the relationship between vascular lesions and ESRD or 50\% reduction in renal function was verified. There was no such correlation in patients treated with RAS blockade, providing indirect evidence that vascular lesions are responsive to RAS blockade therapy.

The limitations of this study are the following. First, this study was in a single center with a short follow-up time. Second, the treatments and clinical variables during follow-up are not in the multivariate Cox model. The therapy and the response to it possibly could confound the correlation between vascular lesions and outcomes. Moreover, it is a retrospective study, and a quite descriptive study. More investigations are required for a better understanding of the correlations we have found.

\section{Conclusion}

Our current study suggests that extra-glomerular vascular lesions are common in IgAN. In addition, the presence of vascular lesions is closely associated with higher MAP and reduced eGFR. Though vascular lesions were not an independent pathological prognostic factor for the primary renal endpoint, their presence still indicates poorer renal outcomes, which may be changed by RAS blockage.

\section{Acknowledgements}

We thank Prof. Qihe Xu for his constructive comments. This work was supported by the National Natural Science Foundation of China (81400715 and U1604186).

\section{Disclosure Statement}

The authors of this manuscript state that they do not have any conflict of interest.

\section{References}

1 Rodrigues JC, Haas M, Reich HN: IgA Nephropathy. Clin J Am Soc Nephrol 2017;12:677-686.

2 Wu J, Chen X, Xie Y, Yamanaka N, Shi S, Wu D, Liu S, Cai G: Characteristics and risk factors of intrarenal arterial lesions in patients with IgA nephropathy. Nephrol Dial Transplant 2005;20:719-727. 


\section{Kidney \\ Blood Pressure Research}

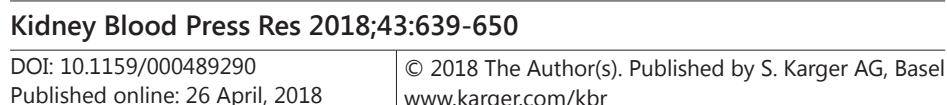

Published onlıne: 26 April, 2018 www.karger.com/kb

Zhang et al.: Arterial Lesions in IgA Nephropathy

- Working Group of the International IgA Nephropathy Network and the Renal Pathology Society: The Oxford classification of IgA nephropathy: pathology definitions, correlations, and reproducibility. Kidney Int 2009;76:546-556.

4 Working Group of the International IgA Nephropathy Network and the Renal Pathology Society: The Oxford classification of IgA nephropathy: rationale, clinicopathological correlations, and classification. Kidney Int 2009;76:534-545.

5 Katafuchi R, Kiyoshi Y, Oh Y, Uesugi N, Ikeda K, Yanase T, Fujimi S: Glomerular score as a prognosticator in IgA nephropathy: its usefulness and limitation. Clin Nephrol 1998;49:1-8.

-6 Berthoux F, Mohey H, Laurent B, Mariat C, Afiani A, Thibaudin L: Predicting the risk for dialysis or death in IgA nephropathy. J Am Soc Nephrol 2011;22:752-761.

7 Goto M, Wakai K, Kawamura T, Ando M, Endoh M, Tomino Y: A scoring system to predict renal outcome in IgA nephropathy: a nationwide 10-year prospective cohort study. Nephrol Dial Transplant 2009;24:30683074.

8 Wakai K, Kawamura T, Endoh M, Kojima M, Tomino Y, Tamakoshi A, Ohno Y, Inaba Y, Sakai H: A scoring system to predict renal outcome in IgA nephropathy: from a nationwide prospective study. Nephrol Dial Transplant 2006;21:2800-2808.

-9 Trimarchi H, Barratt J, Cattran DC, Cook HT, Coppo R, Haas M, Liu ZH, Roberts IS, Yuzawa Y, Zhang H, Feehally J, IgAN Classification Working Group of the International IgA Nephropathy Network and the Renal Pathology Society, Conference Participants: Oxford Classification of IgA nephropathy 2016: an update from the IgA Nephropathy Classification Working Group. Kidney Int 2017;91:1014-1021.

10 Wu LH, Yu F, Tan Y, Qu Z, Chen MH, Wang SX, Liu G, Zhao MH: Inclusion of renal vascular lesions in the 2003 ISN/RPS system for classifying lupus nephritis improves renal outcome predictions. Kidney Int 2013;83:715-723.

-11 Matsushita K, Mahmoodi BK, Woodward M, Emberson JR, Jafar TH, Jee SH, Polkinghorne KR, Shankar A, Smith DH, Tonelli M, Warnock DG, Wen CP, Coresh J, Gansevoort RT, Hemmelgarn BR, Levey AS, Chronic Kidney Disease Prognosis C: Comparison of risk prediction using the CKD-EPI equation and the MDRD study equation for estimated glomerular filtration rate. JAMA 2012;307:1941-1951.

12 Schwartz GJ, Haycock GB, Edelmann CM, Jr., Spitzer A: A simple estimate of glomerular filtration rate in children derived from body length and plasma creatinine. Pediatrics 1976;58:259-263.

13 Shi SF, Wang SX, Jiang L, Lv JC, Liu LJ, Chen YQ, Zhu SN, Liu G, Zou WZ, Zhang H, Wang HY: Pathologic predictors of renal outcome and therapeutic efficacy in IgA nephropathy: validation of the oxford classification. Clin J Am Soc Nephrol 2011;6:2175-2184.

14 El Karoui K, Hill GS, Karras A, Jacquot C, Moulonguet L, Kourilsky O, Fremeaux-Bacchi V, Delahousse M, Duong Van Huyen JP, Loupy A, Bruneval P, Nochy D: A clinicopathologic study of thrombotic microangiopathy in IgA nephropathy. J Am Soc Nephrol 2012;23:137-148.

15 Nasri H, Mubarak M: Significance of vasculopathy in IgA nephropathy patients with regard to Oxford classification and immunostaining findings: a single center experience. J Renal Inj Prev 2013;2:41-45.

16 Chang A, Kowalewska J, Smith KD, Nicosia RF, Alpers CE: A clinicopathologic study of thrombotic microangiopathy in the setting of IgA nephropathy. Clin Nephrol 2006;66:397-404.

$>17$ Brocklebank V, Kavanagh D: Complement C5-inhibiting therapy for the thrombotic microangiopathies: accumulating evidence, but not a panacea. Clin Kidney J 2017;10:600-624.

18 Mathew RO, Nayer A, Asif A: The endothelium as the common denominator in malignant hypertension and thrombotic microangiopathy. J Am Soc Hypertens 2016;10:352-359.

19 Wenzel UO, Bode M, Kohl J, Ehmke H: A pathogenic role of complement in arterial hypertension and hypertensive end organ damage. Am J Physiol Heart Circ Physiol 2017;312:H349-H354.

20 Liu L, Zhang Y, Duan X, Peng Q Liu Q Zhou Y, Quan S, Xing G: C3a, C5a renal expression and their receptors are correlated to severity of IgA nephropathy. J Clin Immunol 2014;34:224-232.

-21 Caliskan Y, Ozluk Y, Celik D, Oztop N, Aksoy A, Ucar AS, Yazici H, Kilicaslan I, Sever MS: The Clinical Significance of Uric Acid and Complement Activation in the Progression of IgA Nephropathy. Kidney Blood Press Res 2016;41:148-157.

22 Le W, Liang S, Hu Y, Deng K, Bao H, Zeng C, Liu Z: Long-term renal survival and related risk factors in patients with IgA nephropathy: results from a cohort of 1155 cases in a Chinese adult population. Nephrol Dial Transplant 2012;27:1479-1485. 


\section{Kidney \\ Blood Pressure Research}

23 Alamartine E, Sauron C, Laurent B, Sury A, Seffert A, Mariat C: The use of the Oxford classification of IgA nephropathy to predict renal survival. Clin J Am Soc Nephrol 2011;6:2384-2388.

-24 Coppo R, Troyanov S, Bellur S, Cattran D, Cook HT, Feehally J, Roberts IS, Morando L, Camilla R, Tesar V, Lunberg S, Gesualdo L, Emma F, Rollino C, Amore A, Praga M, Feriozzi S, Segoloni G, Pani A, Cancarini G, et al.: Validation of the Oxford classification of IgA nephropathy in cohorts with different presentations and treatments. Kidney Int 2014;86:828-836.

-25 Viola J, Soehnlein 0: Atherosclerosis - A matter of unresolved inflammation. Semin Immunol 2015;27:184193.

-26 Gao J, Wei L, Liu X, Wang L, Niu D, Jin T, Yao G, Wang M, Yu Q, Fu R: Association Between IFN-gamma Gene Polymorphisms and IgA Nephropathy in a Chinese Han Population. Kidney Blood Press Res 2017;42:136144.

27 Gao J, Wei L, Fu R, Wei J, Niu D, Wang L, Ge H, Yu Q, Wang M, Liu X, Zhang W: Association of Interleukin-10 Polymorphisms (rs1800872, rs1800871, and rs1800896) with Predisposition to IgA Nephropathy in a Chinese Han Population: A Case-Control Study. Kidney Blood Press Res 2017;42:89-98.

-28 Quek KJ, Ameer OZ, Phillips JK: AT1 Receptor Antagonism Improves Structural, Functional and Biomechanical Properties in Resistance Arteries in a Rodent Chronic Kidney Disease Model. Am J Hypertens 2018; DOI:10.1093/ajh/hpy021.

29 Konishi Y, Nishiyama A, Morikawa T, Kitabayashi C, Shibata M, Hamada M, Kishida M, Hitomi H, Kiyomoto H, Miyashita T, Mori N, Urushihara M, Kobori H, Imanishi M: Relationship between urinary angiotensinogen and salt sensitivity of blood pressure in patients with IgA nephropathy. Hypertension 2011;58:205-211.

-30 Miyake-Ogawa C, Miyazaki M, Abe K, Harada T, Ozono Y, Sakai H, Koji T, Kohno S: Tissue-specific expression of renin-angiotensin system components in IgA nephropathy. Am J Nephrol 2005;25:1-12.

-31 Nishiyama A, Konishi Y, Ohashi N, Morikawa T, Urushihara M, Maeda I, Hamada M, Kishida M, Hitomi H, Shirahashi N, Kobori H, Imanishi M: Urinary angiotensinogen reflects the activity of intrarenal reninangiotensin system in patients with IgA nephropathy. Nephrol Dial Transplant 2011;26:170-177.

-32 Kobori H, Katsurada A, Ozawa Y, Satou R, Miyata K, Hase N, Suzaki Y, Shoji T: Enhanced intrarenal oxidative stress and angiotensinogen in IgA nephropathy patients. Biochem Biophys Res Commun 2007;358:156163. 